\title{
The Relationship between MEG and fMRI
}

A Review Article for the Special Issue of NeuroImage on Multi-Modal Data Fusion

Emma L. Hall, Siân E. Robson, Peter G. Morris and Matthew J. Brookes*

Sir Peter Mansfield Magnetic Resonance Centre, School of Physics and Astronomy, University of Nottingham, UK

* Corresponding author, Dr M J Brookes

Sir Peter Mansfield Magnetic Resonance Centre

University of Nottingham

Nottingham, UK

NG7 2RD

Email: matthew.brookes@nottingham.ac.uk

Tel: +44(0)1159515188 


\begin{abstract}
$\underline{\text { Abstract }}$
In recent years functional neuroimaging techniques such as FMRI, MEG, EEG and PET have provided researchers with a wealth of information on human brain function. However none of these modalities can measure directly either the neuro-electrical or neuro-chemical processes that mediate brain function. This means that metrics directly reflecting brain 'activity' must be inferred from other metrics (e.g. magnetic fields (MEG) or haemodynamics (fMRI)). To overcome this limitation, many studies seek to combine multiple complementary modalities and an excellent example of this is the combination of MEG (which has high temporal resolution) with fMRI (which has high spatial resolution). However, the full potential of multi-modal approaches can only be truly realised in cases where the relationship between metrics is known. In this paper, we explore the relationship between measurements made using fMRI and MEG. We describe the origins of the two signals as well as their relationship to electrophysiology. We review multiple studies that have attempted to characterise the spatial relationship between FMRI and MEG, and we also describe studies that exploit the rich information content of MEG to explore differing relationships between MEG and fMRI across neural oscillatory frequency bands. Monitoring the brain at "rest" has become of significant recent interest to the neuroimaging community and we review recent evidence comparing MEG and fMRI metrics of functional connectivity. A brief discussion of the use of magnetic resonance spectroscopy (MRS) to probe the relationship between MEG/fMRI and neurochemistry is also given. Finally, we highlight future areas of interest and offer some recommendations for the parallel use of fMRI and MEG.
\end{abstract}

Key words: fMRI, MEG, BOLD, neural oscillations, resting state 


\section{Introduction:}

Recent years have seen the field of systems neuroscience revolutionised by functional neuroimaging. Techniques such as positron emission tomography (PET), functional magnetic resonance imaging (fMRI), magnetoencephalography (MEG) and electroencephalography (EEG) combined with sophisticated analysis algorithms now provide researchers with unprecedented information on the mechanisms of human brain function. However, the noninvasive nature of neuroimaging methods means that, in most cases, the precise metric of brain 'activity' that is sought can only be inferred from another, indirect, measurement. For example, in fMRI a change in brain 'activity' is inferred via measurement of local changes in haemodynamics - this necessarily assumes a relationship between 'activity' and the haemodynamic response. In EEG or MEG, measurements of electrical current in the brain are inferred via measurement of extracranial electric (EEG) or magnetic (MEG) field. Here the measurement of interest (neural current) must be estimated based on projection of extracranial field data onto the brain. The indirect nature of assessment of brain 'activity' is a fundamental limitation of non-invasive functional neuroimaging and increasingly, researchers attempt to overcome this issue by combining multiple complementary imaging modalities.

The combination of non-invasive measurements of electrical activity (via MEG or EEG) with fMRI provides an excellent example of multi-modal fusion. fMRI allows characterisation of the spatial signature of brain activity with sub-millimetre spatial resolution (e.g. (Heidemann et al., 2012)). However, the latency and longevity of the haemodynamic response limits temporal resolution to $\sim 5 \mathrm{~s}$. MEG and EEG ostensibly offer more direct insight into electrical brain activity since the measured fields are induced directly from synchronous neuronal current flow. Temporal resolution is limited only by the speed of sampling of the magnetic or electric field (up to $12 \mathrm{kHz}$ in modern MEG systems) and so electrical activity can be characterised on the millisecond timescale at which the brain operates. However, spatial information must be inferred via modelling of extra-cranial field measurements and projection of those measured fields onto the brain; this is a mathematically ill posed problem which limits spatial resolution, and resulting timecourses of neural current typically represent the integrated activity across a relatively large tissue volume. MEG offers a significant advantage compared to EEG in this regard since, unlike the electric field, the magnetic field is not distorted by the inhomogeneous and poorly defined conductivity profile of the brain/skull/scalp, meaning that models of extracranial fields (i.e. forward models) are potentially of greater accuracy in MEG. MEG also offers a higher signal-to-noise ratio and (typically) more scalp based sensors compared to EEG ( 300 compared with typically 64 in EEG). These factors combined give MEG an improved spatial resolution and sensitivity compared to EEG. This said, EEG also offers significant advantages; in 
particular, EEG signals can be measured inside an MRI system, facilitating simultaneous measurements of BOLD and electrophysiology (see e.g. (Gonçalves et al., 2006)). Such measurements offer the potential to investigate, e.g. relationships between the inter-trial variance of BOLD and EEG signals, within single subjects (see e.g. (Mullinger et al., 2013)). This is not (at present) possible with parallel MEG/fMRI experiments where comparisons must be limited to spatial, temporal, cross subject or cross task condition correlation. It is clear therefore that both parallel MEG/fMRI, and simultaneous EEG/fMRI offer complementary advantages/disadvantages to investigate relationships between the BOLD signal and electrophysiology.

For the purpose of the present review, we limit ourselves to the discussion of parallel MEG/fMRI studies. In practice, fusion of MEG and fMRI data is not trivial. Firstly, comparison of MEG and fMRI signals in brain space requires solution of the ill posed inverse problem and this typically introduces a degree of spatial ambiguity. Moreover, even if accurate localisation is possible,the projected signal is rich in information, with time and phase locked event related fields observable alongside neural oscillatory processes (i.e. the magnetic fields generated by rhythmic activity in large neural cell assemblies). Oscillations occur across a wide range of frequencies between 0 and $200 \mathrm{~Hz}$ (commonly labelled $\delta(1-4 \mathrm{~Hz}), \theta(4-8 \mathrm{~Hz}), \alpha(8-13 \mathrm{~Hz}), \beta$ $(13-30 \mathrm{~Hz}), \gamma(>30 \mathrm{~Hz}))$. Within specific brain regions, following a stimulus or task, it is possible to measure changes in oscillatory power, phase and power-phase coupling. In fact, multiple characteristic oscillatory effects are often observed in response to a single event, separated in frequency and/or time. In addition to oscillatory effects, 'evoked' responses are also observed. These typically comprise transient deflections of the measured signal that are time and phase locked to stimulus onset and offset. A good example of the complex neuromagnetic response is the robust effect elicited by a simple finger movement; in the contralateral motor cortex one observes both a decrease in oscillatory power in the beta band and a simultaneous movementrelated increase in power in the gamma band. This is followed by an increase in beta power (above baseline) following movement cessation, known as the post movement beta rebound (PMBR). This type of multifaceted response is typical, and complicates MEG/fMRI data fusion since it remains largely unclear which, if any, MEG detectable effects are driving the fMRI signal. This is a critical point since the fusion of MEG and fMRI to obtain high spatial and temporal resolution is only useful if the two signals reflect directly the same underlying neurophysiological events. With this in mind, recent years have seen the combination of MEG and fMRI open up exciting avenues of research exploring the nature of the haemodynamic signal, the electrophysiological signal, and their relationship to one another. 
In this paper, we will review a body of work on the relationship between MEG and fMRI measurements. Section 2 will describe the origins of both the neuromagnetic and haemodynamic signals and will highlight theoretical and experimental evidence implying that both originate from post synaptic currents. In Section 3 we will discuss some of the key studies aimed at correlating MEG and fMRI measures, placing particular emphasis on neuronal oscillations. Recent years have seen a paradigm shift in fMRI towards approaches which seek to elucidate brain regions exhibiting temporally correlated haemodynamic signals, known as networks of functional connectivity. Current MEG literature has attempted to mirror such findings and in Section 4 we will review the aspects of the MEG signal that appear to correlate with fMRI measures of connectivity. Electrophysiological and haemodynamic effects are both controlled via neurochemical mechanisms, and in Section 5, we will review briefly some of the experimental findings that attempt to correlate both MEG and fMRI to neurotransmitter levels measured using magnetic resonance spectroscopy (MRS). Finally, in Section 6, we will draw these observations together and offer opinions on the relationship between MEG and fMRI, and on the future of parallel studies.

\section{Signal Origins}

The principal origins of both MEG (Cohen, 1968) and blood oxygen level-dependent (BOLD (Ogawa et al., 1990)) fMRI signals are summarised in Figure 1.

\subsection{MEG}

MEG measures the magnetic fields induced above the scalp surface by current flow in the brain. There are two principal sources of current in the brain, post synaptic (dendritic) and axonal; theoretical evidence suggests that the measurable magnetoencephalogram originates largely from post synaptic current flow. There are two reasons for this: 1) dendritic current flow is a good approximation to a current dipole for which field falls with distance as $1 /$ (distance) $^{2}$; whereas axonal currents are quadrupolar and field falls as $1 /$ (distance) $^{3}$. Since MEG detects magnetic field at a relatively large distance, the contribution of dendritic current will be larger. 2) The dipole moment of a single dendrite (approximately 20fAm) is insufficient to generate a detectable extra-cranial magnetic field, as is the quadrupole moment of a single axonal current. In fact, for a detectable field, some tens of thousands of dendritic currents must flow in synchrony. The extended temporal duration of dendritic current (an order of magnitude greater than action potentials (Ahonen et al., 1993)), coupled with the greater abundance of dendrites means that synchrony in dendritic current flow is more likely than in axons. Synchronicity must however be coupled with constructive superposition of the fields from multiple dipoles to generate a measurable signal. This makes stellate cells unlikely to contribute to the measured 
field due to their symmetrically arranged dendrites. It follows that current flow in the dendrites of cortical pyramidal neurons (where dendrites run asymmetrically, and perpendicular to the cortical surface) is the most likely origin of the measurable MEG signals (Ahonen et al., 1993, Hamalainen et al., 1993).

This theoretical argument is supported by simultaneous measurements involving invasive electrophysiology (measured via implanted cortical electrodes) and MEG. Zhu et al. (Zhu et al., 2009) compared multi-unit activity (MUA, which is largely of axonal origin), LFPs (which are thought to derive principally from post synaptic events) and MEG recordings in monkey somatosensory cortex in response to fingertip stimulation. Response latencies measured with the three metrics differed, but MEG and LFP were better matched than MEG and MUA. Further, the MEG and LFP normalised response amplitudes showed no significant difference, and both were significantly larger than MUA, thus supporting the argument that MEG reflects post synaptic events.

\section{2. $B O L D$}

Electrical activity in the brain triggers a complex chain of events which ultimately results in a measurable change in MR signal. The neurochemical processes which accompany electrodynamic changes act on the local vasculature, causing changes in cerebral blood flow, cerebral blood volume, oxygen extraction and metabolism (Belliveau et al., 1991, Kwong et al., 1992, Ogawa et al., 1992). These effects combine to generate changes in relative levels of oxyhaemoglobin and deoxyhaemaglobin and this causes changes in transverse relaxation rate $\left(\mathrm{T}_{2}{ }^{*}\right)$ local to the site of neuronal activity which is the basis of the BOLD fMRI signal (Ogawa et al., 1990). This transient increase in MR signal is usually termed the BOLD haemodynamic response function (HRF). The haemodynamic parameters mentioned above are thought to be very tightly spatially coupled to electrodynamics which gives BOLD its excellent spatial resolution. However, it takes several seconds for the HRF to peak following stimulus onset and likewise several seconds to return to baseline following stimulus cessation; further, the precise timing varies across subjects and brain regions (Handwerker et al., 2004). This gives BOLD poor temporal resolution that is limited by the physiology of the response itself and not by limitations of hardware.

The convoluted dependence of the BOLD signal on haemodynamic and neurovascular coupling parameters means that it cannot be viewed as a quantitative metric of brain activity. A change in the BOLD signal could arise from a change in neuronal activity, neurovascular coupling, haemodynamic state or a combination of these. In addition, the BOLD response is affected by 
measurement parameters including magnetic field strength, echo time (TE), repetition time (TR) and the imaging sequence used. Previous studies have shown that the complex dependency of BOLD on neurovascular coupling can cause unexpected results, for example if basal blood flow is very high, the BOLD change to a stimulus may be lost (Gauthier et al., 2011). fMRI is also very susceptible to physiological noise, for example studies have shown that both the cardiac and respiratory cycles generate BOLD artifacts (e.g. (Dagli et al., 1999, Birn et al., 2006)). Despite its qualitative nature, it is generally accepted that the BOLD response is, in effect, an index of energy demand in the brain, with a positive response indicating increased energy usage due to local cortical processing. In considering the relationship between MEG and BOLD, it is therefore instructive to consider the physiological origins of this energy demand.

Attwell and Laughlin (Attwell and Laughlin, 2001) estimated that in human grey matter approximately $74 \%$ of ATP consumption was associated with repolarisation following synaptic currents, and that energy demand is not dominated by spiking activity (Cruetzfeldt, 1975). This calculation is in general agreement with experiments based upon simultaneous recording of electrophysiology and BOLD. Combined electrophysiology and fMRI is relatively popular to explore the coupling between the neuronal activity and haemodynamic response, although most literature is limited to the study of experimental animals (Logothetis et al., 2001, Shmuel et al., 2006, Goense and Logothetis, 2008, Goloshevsky et al., 2008). Multiple studies have demonstrated that the BOLD response is more closely coupled to LFP rather than MUA (Logothetis et al., 2001, Niessing et al., 2005, Shmuel et al., 2006, Goense and Logothetis, 2008). In addition, Logothetis and colleagues (Logothetis et al., 2001) showed that spiking activity was not a good indicator of the BOLD response and linearity was seen between BOLD and LFP responses. Shmuel and colleagues (Shmuel et al., 2006) found correlation between negative BOLD responses and a reduction in neuronal activity measured using LFP in the visual cortex of monkeys. Additionally, linearity between BOLD and somatosensory evoked potentials due to rat forepaw stimulation has been demonstrated (Goloshevsky et al., 2008). Evaluation of these relationships in humans is difficult due to the invasive procedure; however invasive recordings are occasionally undertaken when patients undergo surgery and studies in general further imply a close relationship between LFP and BOLD (Conner et al., 2011, Harvey et al., 2013). Conner et al (Conner et al., 2011) investigated the spatial variation of LFP/BOLD coupling using electrocorticography. LFP-BOLD coupling was dependant on frequency band and brain region but generally, positive correlations were seen in the gamma band, and negative correlation in the beta band. This general pattern was also shown by Mukamel et al. (Mukamel et al., 2005). 
The generally close relationship between BOLD and LFP, which is predominantly post synaptic in origin, is critical in the context of the present paper since it shows that fMRI signals, like MEG signals, are thought to largely result from a dendritic (rather than axonal) origin. The combination and/or comparison of MEG and fMRI therefore make theoretical sense.

\section{Task driven studies using MEG and fMRI:}

The fact that MEG and fMRI signals are likely to originate from similar underlying physiological processes suggests that, despite the two signals exhibiting fundamentally different properties (Figure 1), a close relationship is highly probable. In this section we examine two principal modes of comparison between MEG and fMRI; spatial similarities and temporal/spectral relationships.

\subsection{Spatial Relationships}

The spatial relationship between brain areas identified as exhibiting a task induced signal change by BOLD fMRI and MEG is of significant interest, particularly in applications such as presurgical planning (Grummich et al., 2006) where spatial precision is paramount. However, the question of whether BOLD and neuromagnetic signals originate from the same brain location is, to an extent, confounded by the limits of spatial resolution, and the differences between MEG and fMRI.

In MEG, the spatial signature of changes in neural current across the cortex is inferred via projection of magnetic field data into brain space. This projection forms a mathematically illposed problem (the MEG inverse problem) and this places a fundamental limit on the spatial accuracy of MEG. Over the last two decades there have been significant advances in this field of research, which have been key to providing insights into the spatial relationship between MEG and fMRI. There are many algorithms available for MEG source estimation, with examples including Minimum Norm (Hämäläinen and Ilmoniemi, 1994), LORETA (Pascual-Marqui et al., 1994), FOCUSS (Gorodnitsky et al., 1995), MUSIC (Mosher et al., 1992), beamforming (Robinson and Vrba, 1998, Sekihara et al., 2001), CHAMPAGNE (Wipf et al., 2010) and many others. The similarities and differences of these reconstruction techniques have been discussed elsewhere (e.g. (Vrba and Robinson, 2001, Hamalainen and Hari, 2002, Singh, 2006)) and it is out of the scope of this article to provide an extensive review of these techniques. However it is critical to note that, in general, the ill-posed nature of the problem requires that a specific set of 
assumptions is made prior to projection, meaning that the derived spatial signature likely depends on the methodology used for source reconstruction. For example, beamforming (Van Drongelen et al., 1996, Van Veen et al., 1997, Robinson and Vrba, 1998, Gross et al., 2001a, Sekihara et al., 2001) is known to suppress and/or mis-localise spatially separate sources that exhibit high temporal correlation; while minimum norm reconstructions (Hämäläinen and Ilmoniemi, 1994) can lead to inaccuracies in radial depth estimation. In addition, most algorithms employ a point dipole model of cortical current, which is an excellent approximation in the case where a small volume of tissue is generating the measured field; however, this model breaks down for coherent activity across large tissue volumes and this can limit spatial accuracy. A further problem for source reconstruction originates from the rich nature of the MEG signal: As described above, there are multiple aspects to the MEG signal that all have different signal to noise ratios (SNRs) and potentially different spatial origins. The spatial accuracy in reconstructing these aspects is not necessarily comparable. For example, a reconstructed gamma band signal is not necessarily equivalent in spatial accuracy to a reconstructed beta band signal, even in the same subject, due to differences in SNR between the two effects. Likewise, different signal features may not necessarily adhere to the same assumption set (e.g. beamforming may be effective in reconstructing neural oscillations, but the assumption regarding temporal correlation may break down for evoked signals). It therefore follows that judicious selection and appropriate understanding of the projection algorithm is critical if an unbiased view of spatial similarities and/or differences between MEG and fMRI is to be generated.

It is tempting to ascribe differences between localisation of fMRI and MEG signals solely to inaccuracies in MEG forward and inverse models; however we should point out that errors in localisation can arise from other sources. Spatial comparison necessarily requires coregistration of MEG and fMRI functional data to the same anatomical coordinate space. This requires coregistration of fMRI data to an anatomical image and accurate knowledge of the MEG sensor geometry relative to the brain anatomy. The former can be problematic due to differences in MR sequences between the functional acquisition and the anatomical image commonly used for overlaying results. Specifically, echo planar images typically used for fMRI are low bandwidth and therefore highly susceptible to spatial distortion and/or dropout caused by field inhomogeneity and gradient non-linearity. MEG data are co-registered to the brain anatomy via the use of fiducial markers. Usually, markers comprise electromagnetic coils which are localised relative to the MEG system by energising them and modelling the resultant fields. They are subsequently localised relative to the head surface, either by digitisation and surface matching (Adjamian et al., 2004), or by MR acquisition - replacing the coils with MR visible markers. In 
both cases significant errors can result, from movement of the markers on the skin, inaccuracies in digitisation, or susceptibility artifacts giving a systematic error in MRI. In addition to these experimental errors, the BOLD response itself can demonstrate mislocalisation of the actual site of neuronal activity: for example pooled oxygenated blood in large veins may yield a significant BOLD response distal to the true site of neuronal activity (a particular problem at low field) (Bandettini and Wong, 1997). Taken together, the above evidence implies that a high degree of ambiguity is involved in spatial comparisons between fMRI and MEG.

Despite the difficulties, there have been a number of efforts to examine the co-localisation of MEG and BOLD signals. Indeed, a wide range of disparate MEG-derived signals have demonstrated a close spatial relationship with BOLD, including task induced changes in oscillatory power in multiple frequency bands from theta to gamma, as well as the phase locked evoked response (Moradi et al., 2003, Brookes et al., 2005, Winterer et al., 2007, Muthukumaraswamy and Singh, 2008, Zumer et al., 2010, Stevenson et al., 2011, Stevenson et al., 2012). For example, Singh and colleagues (Singh et al., 2002) measured the spatial signature of BOLD and induced oscillatory power changes in MEG in response to two cognitive tasks, showing a good spatial relationship between BOLD and the focal locations of event related power changes in alpha band signals. The principal results of this study are reproduced in Figure 2 which shows the group fMRI and MEG beamformer maps for a letter fluency task. Table 1 summarises further studies reporting spatial discrepancies (measured as Euclidian distance) between peaks in MEG and BOLD responses. All studies demonstrate a reasonably close spatial relationship, with mismatches of $<30 \mathrm{~mm}$. Although spatial discrepancies of this order are substantial, they cannot easily be attributed to a fundamental difference in signal origins, given the limitations in accuracy of the techniques employed, as described above.

The evidence from using MEG and fMRI independently to assess Euclidean distance between sources therefore implies that spatial relationships exist between these modalities. Other studies go beyond this, and use fMRI spatial information to inform the reconstruction of MEG data (Dale et al., 2000, Fujimaki et al., 2002, Ahlfors and Simpson, 2004, Babiloni et al., 2004, Sato et al., 2004, Im et al., 2005, Henson et al., 2010, Ou et al., 2010). For example Henson and colleagues use a parametric empirical Bayesian framework for fMRI constrained MEG source reconstruction. This enables the use of fMRI based priors to solve the MEG inverse problem. Interestingly, the authors found higher model evidence when employing fMRI priors to inform a minimum norm solution, implying a degree of spatial concordance. (However, the same study found no such improvement when using a multiple sparse priors (MSP) inverse model). Most importantly, this study and others like it exemplify more principled and statistically rigorous 
frameworks in which to test the existence of significant spatial relationships between MEG and fMRI. This said, studies aimed at data fusion in this way must still bear in mind the potential for systematic errors in localisation, as described above.

\subsection{Temporal and Spectral relationships}

Given the difficulties in relying only on spatial aspects of task induced fMRI and MEG signals to compare modalities, it is instructive to include temporal and spectral analyses to provide insight into the aspects of electrophysiology that are most closely aligned with the BOLD response. An example is provided by (Zumer et al., 2010) who examine the correlation between temporally deconvolved BOLD responses and the MEG response to a visual stimulus across a wide range of frequency bands. This study (the main results from which are given in Figure $3 \mathrm{~A} / \mathrm{C}$ ) summarises a general finding that a high degree of negative correlation is observed between stimulus induced BOLD responses and induced changes in neuronal oscillatory amplitude in the low frequency bands (e.g. alpha and beta). This low frequency negative correlation is complemented by a significant positive correlation in the mid and high gamma range. MEG results bear distinct similarities to results acquired using invasive electrode recording of LFP signals in the auditory cortex of conscious epilepsy patients during natural auditory stimulation. These results, published by Mukamel and colleagues (Mukamel et al., 2005), are captured in Figure 3B for comparison. Notice the general similarities between MEG and LFP, with negative correlation at low frequency and positive correlation at high frequency. This change in polarity may reflect a difference in functionality, e.g. low frequency responses may represent long range thalamocortical or cortico-cortical interaction whereas high frequency responses more likely represent localised networks. It is interesting to note also the differences in the curves in Figures $3 \mathrm{~A}$ and B: the fall in correlation in MEG at high frequency is likely to relate to the loss of SNR. The difference in the frequency at which a cross over between negative and positive correlation is observed $(\sim 50 \mathrm{~Hz}$ for $\mathrm{MEG}$ and $\sim 25 \mathrm{~Hz}$ for LFP) could result from many factors including differences between LFP and MEG, differences between cortical areas or stimulation type. Nevertheless these results show direct parallels between MEG and LFP, and also the relationship of both to fMRI.

There are a number of studies that have attempted to further probe the relationship between MEG and BOLD via parametric variation of stimulus parameters. E.g. Stevenson and colleagues (Stevenson et al., 2011) investigate BOLD and $\beta$-band responses to graded visual stimuli (i.e. variation of Michelson contrast), showing that BOLD signal increases non-linearly with contrast (see also (Tootell et al., 1995)) whilst beta band power loss during stimulation was approximately equal regardless of visual contrast. This was in contrast to the visual gamma 
band response, which increased monotonically with stimulus contrast, (see also (Hall et al., 2005)). Such findings have led to a hypothesis that the BOLD response may comprise a more direct reflection of gamma oscillations than of lower frequency effects (Logothetis et al., 2001, Hall et al., 2005, Niessing et al., 2005). However, MEG based evidence is beginning to show that significant demarcation exists between BOLD and gamma oscillations; for example recent work (Muthukumaraswamy and Singh, 2008, 2009) reported that gamma amplitude in primary visual cortex exhibits substantial variation with spatial frequency, whereas BOLD responses do not. Likewise, further work (Swettenham et al., 2013) shows that unlike visual gamma oscillations, BOLD responses are relatively insensitive to stimulus parameters such as colour. Overall, evidence suggests that the relationship between fMRI and neural oscillations (at least in visual cortex) is a complex one. It is unlikely that a simple one-to-one relationship exists between haemodynamics and oscillations in any individual frequency band (Winterer et al., 2007) and further experimentation is required to better elucidate existing relationships.

\section{Functional Connectivity Approaches}

\subsection{Functional Connectivity MRI}

In recent years there has been a paradigm shift in fMRI analysis with the increased use of "functional connectivity" approaches, the aim being to show that spatially separate regions demonstrate a 'functional relationship', usually measured as a statistical interdependency between signals. Functional connectivity, assessed by fMRI (fcMRI), typically examines temporal correlation between BOLD signals from separate regions. This is often done with the brain apparently at rest (i.e. in the absence of a defined task). Biswal et al. (Biswal et al., 1995) found that left and right motor cortices demonstrate correlated BOLD timecourses in the absence of a stimulus. This early work initiated a large number of studies which have led to the discovery that the brain can be divided into to a relatively small number of highly robust spatially welldefined networks of connectivity (Beckmann et al., 2005, Damoiseaux et al., 2006) some associated with sensory function and others with cognitive or attentional processes. Functional connectivity between brain regions is now generally accepted as being key to healthy brain function; moreover, abnormalities in connectivity have been observed in a number of pathological cases (Tian et al., 2006, Seeley et al., 2009, Liu et al., 2013a, Liu et al., 2013b). The current use of fcMRI is now wide-ranging, from clinical studies in Alzheimer's disease (Liu et al., 2013b) and Parkinson's disease (Liu et al., 2013a), to the study of neurodevelopment (Jolles et al., 2011) and the effect of pharmacological agents (Liao et al., 2012). Indeed, this area of neuroimaging research has grown dramatically of late, with publications increasing four-fold in the past four years (source PubMED, publications in 2008 and 2012, functional connectivity in the title/abstract and MRI featured). 
The robust and spatially distinct nature of measured networks of functional connectivity provides a new platform upon which to investigate relationships between fMRI and MEG. This platform ameliorates some of the confounds associated with spatial comparisons listed above: for example, spatial comparisons can be made not just between unimodal regions measured across modalities, but between whole brain networks, each comprising multiple nodes. Furthermore, comparisons can be made over multiple networks within a single study. Most importantly, cross modality studies of functional connectivity provide novel information on the electrophysiological processes that underpin temporal correlation between BOLD timecourses, and therefore should provide insights into the electrophysiological correlates of BOLD signals.

\subsection{Studies of MEG Measures of Connectivity}

The utility of MEG to measure connectivity has been shown by a large number of previous studies (examples include (Tass et al., 1998, Ioannides et al., 2000, Gross et al., 2001a, Gross et al., 2002, Nolte et al., 2004a, Ramnania et al., 2004, Schlögl and Supp, 2006, Jerbi et al., 2007, Gow et al., 2008, Nolte et al., 2008, Schoffelen and Gross, 2009)). However, the use of MEG to investigate the large scale distributed networks often observed in fMRI is relatively recent, with studies focussed on confirming that such networks are electrophysiological in origin, and are not a haemodynamic artefact (e.g. arising from connected vascular territories). Functional connectivity metrics for MEG are complicated by the richness of the MEG signal. In fcMRI, functional connectivity assessment is largely confined to correlation between BOLD timecourses; however, in MEG there are many potential metrics for interaction (Schölvinck et al., 2013). Coupling between band limited oscillatory power envelopes is perhaps the simplest metric and has been used successfully to elucidate long range statistical dependency between MEG signals. For example, Liu and colleagues (Liu et al., 2010) demonstrated cross hemisphere beta band power correlations between MEG sensor measurements. Coupling between oscillatory phase has also been employed; for example, Gross et al. (Gross et al., 2001b) proposed a popular method to assess coherence between cortical sources and this has been used to identify networks of connectivity in Parkinsonian patients (Timmermann et al., 2002). There are also many other ways to image connectivity including phase lag index (Stam et al., 2007, Hillebrand et al., 2012), imaginary coherence (Nolte et al., 2004b, Sekihara et al., 2011), synchronisation likelihood (Stam and van Dijk, 2002) and others. It is beyond the scope of this review to list all of the possible metrics for functional coupling in MEG; however this abbreviated list provides some indication of the myriad metrics that are available.

\subsection{Relationship between fMRI and MEG: Measures of Functional Connectivity}


The relationship between the BOLD response and changes in MEG oscillatory power during a task implies that if the large scale distributed networks observable in fMRI were to be measured in MEG, then assessment of band limited power correlation would be informative. This has been shown to be the case, and there is now good evidence emerging showing that significant statistical interdependencies exist between source reconstructed MEG signals at spatially separate nodes of fMRI networks. Specifically, if one takes the band limited amplitude envelope of MEG data at some seed location within a node of an fMRI network, one generally finds that across the whole brain, the highest correlation often occurs within a separate node of that same fMRI network. One of the first demonstrations of this (de Pasquale et al., 2010) used source space reconstruction and correlation between band limited power envelopes in the theta (3.5$7 \mathrm{~Hz})$, alpha $(8-13 \mathrm{~Hz})$, beta $(14-25 \mathrm{~Hz})$, gamma $(27-70 \mathrm{~Hz})$ and broad $(1-150 \mathrm{~Hz})$ frequency bands. They found networks in MEG that demonstrated similarities with default mode and dorsal attention networks commonly found in fMRI. Further expansion of these findings came from Brookes et al. (Brookes et al., 2011a) who used a seed based correlation approach to show that temporally down-sampled amplitude envelopes are correlated across the left-right motor cortices, mirroring the findings of Biswal and colleagues (Biswal et al., 1995). Dependence on frequency band was also investigated with the strongest correlation evident in the beta band. These findings were also mirrored by Hipp et al. (Hipp et al., 2012), who have extended this to auditory and visual networks, as well as attentional networks that have been previously well characterised by fMRI. Analogous to the progression of analysis techniques in fcMRI, independent component analysis (ICA) has been developed as a way of identifying networks in MEG data (Brookes et al., 2011b). Again, the down-sampled Hilbert envelope was used as an input to a temporal ICA analysis framework. This allowed identification of several temporally independent components, which were known to exist within clusters of voxels that form distributed spatial patterns. These patterns exhibit significantly higher spatial correlation to BOLD networks than matched surrogate data, implying a degree of spatial concordance for some networks. This ICA approach has also been applied to task positive data (Brookes et al., 2012, Luckhoo et al., 2012, Hall et al., 2013).

Evidence therefore suggests that a degree of spatial similarity exists between patterns of haemodynamic and electrophysiological connectivity. Networks observed in MEG are not perfect spatial matches to those observed in fMRI and the differences, which are often overlooked, may be just as important as the similarities. One example of such differences is the sensorimotor network, where the supplementary motor area (SMA) displays high correlation with left and right motor cortices in fMRI but is rarely seen in MEG. This discrepancy could reflect a genuine difference between the sensorimotor network observed in fMRI and MEG due 
to the different signal origins. However, it could also relate to technical limitations of MEG not being able to accurately reconstruct the sources present in the SMA. Indeed, although network metrics offer some advantages over spatial matching of task dependent regions, spatial matching of networks remains confounded because the spatial smoothness and (more generally) the ill posed inverse problem necessarily generates spatial imprecision.

One interesting aspect of these investigations is the finding that the best spatial match between fMRI and MEG networks occurs, in many cases, via assessment of neural oscillations in the beta frequency band (Figure 4B). This finding should be treated somewhat cautiously, since it is well known that the SNR of MEG data falls with increasing frequency; furthermore, the high gamma band can be affected by muscle artifacts (see (Hall et al., 2013) for specific examples of this). In the resting state, data are necessarily unaveraged and thus SNR and muscle artifacts are more likely to have an effect. It is therefore possible that the preference for beta band in connectivity analyses may be brought about by increased signal to noise in this frequency range. However, this caveat aside, the finding of frequency dependence in MEG functional connectivity analyses may offer useful insights into the relationship between fMRI and MEG, and more importantly the fundamental role of neural oscillations in the brain.

An increasing body of literature suggests that neural oscillations might perform a key role in binding separate brain regions together and promote information transfer between distant foci (Engel and Singer, 2001, Buzsáki and Draguhn, 2004, Roopun et al., 2008). Moreover, a model has been proposed (Donner and Siegel, 2011) in which decreased beta/increased gamma amplitude is suggested to represent local neural processing, whereas increased beta power is associated with 'integrative processes' across larger networks. This model is in agreement with the general finding in task positive studies that local increases in activity (as evidenced by a positive BOLD response) are associated with increased gamma amplitude and decreased low frequency (including beta) amplitude (see Figure 3). Further, the model may also explain the finding that the closest MEG correlate to functional connectivity between networks occurs in the beta band (Figure 4). This model is therefore supported by much of the available evidence from traditional (task based) comparisons of fMRI and MEG as well as more recent functional connectivity comparisons. It offers the exciting possibility to unite these two areas, and ultimately to generate a framework against which future multi-modal studies can combine data from fMRI and MEG in a principled way. 


\section{Relationship Between Neurotransmitters and fMRI/MEG:}

Neuronal activity is mediated by a delicate balance of excitatory and inhibitory neurotransmitters. Aspects of this neurotransmission can be measured using magnetic resonance spectroscopy and it is informative, in order to further understand the origins of MEG and BOLD, to review briefly this literature.

\subsection{MEG and MRS Studies}

Studies relating MEG responses to neurotransmitters have focussed mainly on gammaaminobutyric acid (GABA) (Edden et al., 2009, Muthukumaraswamy et al., 2009, Gaetz et al., 2011, Muthukumaraswamy et al., 2013). Muthukumaraswamy et al. (Muthukumaraswamy et al., 2009) found a significant positive correlation between peak frequency of induced gamma band oscillations in visual cortex (in response to a stationary grating) and baseline GABA concentration in the occipital lobe (Figure 5). This relationship was also shown by (Edden et al., 2009). Gaetz and colleagues (Gaetz et al., 2011) reported similar effects in the motor system: participants performed a button press task and the frequency of gamma oscillations was positively correlated with GABA/NAA. In addition, the amplitude of the PMBR was positively correlated with GABA/NAA. These results were said to support the theory that the PMBR is a period of GABAergic inhibition. Other studies have used pharmacological intervention to manipulate endogenous GABA levels in order to observe the effects on MEG signals (Hall et al., 2010, Hall et al., 2011, Muthukumaraswamy et al., 2013). Diazepam is a GABA-A receptor modulator and has been shown to have a cross-frequency impact on MEG power (Hall et al., 2010) and to increase the beta band power loss during movement, but leave PMBR and movement related gamma increase unchanged in response to finger movement (relative to predrug baseline) (Hall et al., 2011). Tiagabine has also been used to increase the synaptic GABA concentration by blockade of the GAT1 uptake receptor (Muthukumaraswamy et al., 2013). Tiagabine induced an elevated baseline beta power; increased movement related beta decrease and reduced PMBR in response to finger movement (relative to pre movement baseline). These results, taken collectively, begin to elucidate a neurochemical mechanism underlying neural oscillations, with combined evidence pointing towards beta oscillations as a good indicator of GABAergic inhibition, at least in the motor system.

\subsection{BOLD and MRS Studies}

A greater number of studies investigate the relationship between BOLD and neurotransmitter levels. Chen and colleagues (Chen et al., 2005) demonstrated that elevated endogenous GABA levels, elicited using vigabatrin in rats, reduced stimulus induced BOLD responses to forepaw stimulation. In humans, several studies have looked at the correlation of GABA with positive 
BOLD responses. For example Muthukumaraswamy and co-workers (Muthukumaraswamy et al., 2009) measured BOLD responses to a static grating showing that BOLD change was negatively correlated with GABA concentration (Figure 5). Donahue et al. (Donahue et al., 2010) looked at BOLD, CBV and CBF responses to an $8 \mathrm{~Hz}$ reversing checkerboard and baseline GABA in visual cortex. Significant negative correlation was seen between the BOLD signal change and GABA/Cr ratio, in agreement with Muthukumaraswamy. A separate study (Northoff et al., 2007) found that resting GABA measurements from the anterior cingulate cortex (ACC) correlated with negative BOLD responses in the ACC caused by affective picture judgement and perception. A more recent study (Muthukumaraswamy et al., 2012) looked at the haemodynamic response function obtained from a static visual grating paradigm and its relationship with basal GABA concentration. The amplitude of the HRF was shown to be negatively correlated with GABA concentration, and the HRF width was positively correlated. The change in HRF shape is suggestive of a change in the neurovascular coupling rather than a neuronal source. These studies support a logical conclusion that increased GABAergic inhibition results in decreased BOLD response magnitude.

Relatively few studies have assessed the role of glutamate in the BOLD response to a stimulus (Duncan et al., 2011, Falkenberg et al., 2012). Duncan et al. (Duncan et al., 2011) found that glutamate levels in the perigenual ACC were positively correlated with BOLD signal change in the supragenual ACC. Falkenberg and colleagues (Falkenberg et al., 2012) measured basal glutamate in the left and right dorsal ACC and BOLD during an auditory cognitive control task. The BOLD response as a function of task difficulty depended on basal glutamate levels: low glutamate levels led to increased BOLD response with task difficulty, but subjects with high glutamate levels demonstrated higher BOLD responses for the easier tasks across many brain regions. Again these studies support the notion that increased glutamate, in general, leads to increased BOLD responses. Future studies are now required to understand how glutamatergic neurotransmission affects MEG signals; such studies will likely facilitate further insight into relationships between MEG and fMRI.

\section{CONCLUSIONS}

For many researchers, multi-modal imaging using MEG and fMRI in parallel offers the obvious and exciting possibility of measurement of neurophysiological processes with excellent spatial resolution provided by fMRI, and excellent temporal resolution provided by MEG. The ability to track neural dynamics which such precise resolution may reflect a 'holy grail' of functional neuroimaging; however, we would argue that a direct combination of fMRI and MEG signals is only useful if 1) we have good evidence that the two modalities reflect the same underlying 
neurophysiological process and 2) a physical framework by which to combine information from the two techniques is available. Such a framework can only be the result of a good understanding of the relationship between the measured signals and for this reason, the primary purpose of this article was to review current evidence relating to that relationship.

We have presented both theoretical and experimental evidence showing that both the measured MEG and fMRI signals are primarily driven by post synaptic (or dendritic) cellular processes. However, MEG and fMRI signals are disparate in nature, being reflective of electrophysiology and haemodynamics respectively. Their relationship is further complicated by a fundamental lack of understanding as to the role of the measurable electrophysiological signals as well as the neurovascular coupling that mediates the BOLD response. That said, collective evidence from multi-modal studies across over a decade of research is beginning to generate a picture that may offer insight, not only into the relationship between fMRI and MEG, but rather into the fundamental role of many of the measurable processes themselves.

In this paper we have reviewed evidence that suggests spatial concordance between the BOLD response and MEG metrics including oscillations and evoked responses. These spatial relations have been shown to exist using 'traditional' analyses (measuring of task related change in unimodal brain areas) and also using functional connectivity approaches. Aside from demonstrating the expected (given the origin of the two signals) spatial agreement, these observations, coupled with a cross spectral assessment, offer more subtle insight into how different aspects of the MEG signal relate to the haemodynamic response. For instance, assessment of correlation between stimulus induced responses in unimodal brain areas shows evidence for a general trend towards negative correlation between BOLD and low frequency neural oscillations, and a concomitant positive correlation between BOLD and high frequency oscillations. Although we must stress that, riding on top of this trend are subtle disparities; e.g. parametric variation of stimulus parameters having different effects on gamma and BOLD signals, the general trend would appear to fit with the model of Donner and Siegel suggesting that an increase in local processing is signified by an increase in gamma oscillations and a decrease in beta oscillations. Further, we have seen evidence from functional connectivity studies potentially suggesting the involvement of beta band oscillatory processes in the large scale functional connectivity network patterns that are observed in BOLD fMRI. This potentially suggests a role for beta band oscillations in information transfer between spatially separate nodes of known cortical networks: it fits with the notion that neural oscillations are involved in long range communication, and again is in agreement with a model suggesting positive beta amplitude may be indicative of integrative processes across distal brain regions. 
The relationship between BOLD and MEG signals is undoubtedly complex and the observations and arguments presented here remain far from a quantitative understanding. However, we are now in a position to generate testable hypotheses. Furthermore, the richness of the MEG signal is constantly being exploited with novel metrics continually being devised. For example, imaging of signal complexity or entropy (Robinson et al., 2013) offers a potential new way to provide further insight into the relationships between neuroimaging signals.

Parallel MEG/fMRI studies such as those summarised here have generated a wealth of information. However, it is becoming increasingly clear that input from other related measurements is necessary if further direct insight is to be gained into the basis of MEG and fMRI signals. In this context, some of the most important evidence to date has derived from the growing literature on combining MEG and/or fMRI with MRS. Recent studies have begun to elucidate how basal levels of GABA and glutamate covary with haemodynamic and electrophysiological signals, shedding potential light on the aspects of neurochemistry that drive functional signals. However, particularly in the case of glutamate, these basal proton MRS metrics may not be good measures of neurotransmitter pools, still less of neurotransmitter activity. Future studies using carbon-13 MRS may help in this regard, as they enable the rates of neurotransmitter cycling (glutamate/glutamine and GABA/glutamate/glutamine) to be determined and these are much better estimators of excitatory and inhibitory neurotransmitter activity.

For many, the key question is "which technique should I use for my study - MEG or fMRI?" To this we arrive at the rather obvious answer that, in studies directed towards precise spatial characterisation of brain regions or networks, BOLD is a better alternative, despite its indirect nature (one caveat being that in patient groups where the local vasculature is significantly perturbed, BOLD can be misleading (see for example (Rossini et al., 2004)). For high temporal resolution, MEG is preferable. However, the true power of neuroimaging techniques to provide new information on brain function and dysfunction lies not in their individual use, but rather their integration. Such integration must be aimed beyond simply gaining high temporal resolution from MEG and high spatial resolution from fMRI but rather towards exploiting the relative advantages of these techniques and others (MRS, concurrent EEG/fMRI). At present, functional neuroimaging offers a wealth of non-invasive methods to probe aspects of brain function including haemodynamics (fMRI), electrophysiology (MEG/EEG), and neurochemistry (MRS). It is by combining these technologies within carefully designed experimental paradigms 
that we may test current models relating to the signals we measure, and ultimately realise the potential of functional imaging for genuine insight into functional brain physiology.

\section{Acknowledgements}

We acknowledge the Leverhulme Trust for fellowship support of MJB. We also acknowledge the Medical Research Council, the University of Nottingham and the Dr Hadwen Trust for funding. The Dr Hadwen Trust for Humane Research (DHT) is the UK leading medical research charity that funds and promotes exclusively human-relevant research that encourages the progress of medicine with the replacement of the use of animals in research.

\section{References}

Adjamian P, Barnes GR, Hillebrand A, Holliday IE, Singh KD, Furlong PL, Harrington E, Barclay CW, Route PJG (2004) Co-registration of magnetoencephalography with magnetic resonance imaging using bite-bar-based fiducials and surface-matching. Clinical Neurophysiology 115:691-698.

Ahlfors SP, Simpson GV (2004) Geometrical interpretation of fMRI-guided MEG/EEG inverse estimates. NeuroImage 22:323-332.

Ahonen AI, Hamalainen MS, Kajola MJ, Knuutila JET, Laine PP, Lounasmaa OV, Parkkonen LT, Simola JT, Tesche CD (1993) 122-channel squid instrument for investigating the magnetic signals from the human brain. Phys Scr.

Attwell D, Laughlin SB (2001) An Energy Budget for Signaling in the Grey Matter of the Brain. J Cereb Blood Flow Metab 21:1133-1145.

Babiloni F, Mattia D, Babiloni C, Astolfi L, Salinari S, Basilisco A, Rossini PM, Marciani MG, Cincotti F (2004) Multimodal integration of EEG, MEG and fMRI data for the solution of the neuroimage puzzle. Magnetic Resonance Imaging 22:1471-1476.

Bandettini PA, Wong EC (1997) A hypercapnia-based normalization method for improved spatial localization of human brain activation with fMRI. NMR in Biomedicine 10:197203.

Beckmann CF, DeLuca M, Devlin JT, Smith SM (2005) Investigations into resting-state connectivity using independent component analysis. Philosophical Transactions of the Royal Society B: Biological Sciences 360:1001-1013.

Belliveau JW, Kennedy DN, McKinstry RC, Buchbinder BR, Weisskoff RM, Cohen MS, Vevea JM, Brady TJ, Rosen BR (1991) FUNCTIONAL MAPPING OF THE HUMAN VISUAL-CORTEX BY MAGNETIC-RESONANCE-IMAGING. Science 254:716-719.

Birn RM, Diamond JB, Smith MA, Bandettini PA (2006) Separating respiratory-variation-related fluctuations from neuronal-activity-related fluctuations in fMRI. NeuroImage 31:15361548.

Biswal B, Zerrin Yetkin F, Haughton VM, Hyde JS (1995) Functional connectivity in the motor cortex of resting human brain using echo-planar mri. Magnetic Resonance in Medicine 34:537-541.

Brookes MJ, Gibson AM, Hall SD, Furlong PL, Barnes GR, Hillebrand A, Singh KD, Holliday IE, Francis ST, Morris PG (2005) GLM-beamformer method demonstrates stationary field, alpha ERD and gamma ERS co-localisation with fMRI BOLD response in visual cortex. NeuroImage 26:302-308.

Brookes MJ, Hale JR, Zumer JM, Stevenson CM, Francis ST, Barnes GR, Owen JP, Morris PG, Nagarajan SS (2011a) Measuring functional connectivity using MEG: Methodology and comparison with fcMRI. NeuroImage 56:1082-1104. 
Brookes MJ, Liddle EB, Hale JR, Woolrich MW, Luckhoo H, Liddle PF, Morris PG (2012) Task induced modulation of neural oscillations in electrophysiological brain networks. NeuroImage 63:1918-1930.

Brookes MJ, Woolrich M, Luckhoo H, Price D, Hale JR, Stephenson MC, Barnes GR, Smith SM, Morris PG (2011b) Investigating the electrophysiological basis of resting state networks using magnetoencephalography. Proceedings of the National Academy of Sciences 108:16783-16788.

Buzsáki G, Draguhn A (2004) Neuronal Oscillations in Cortical Networks. Science 304:19261929.

Chen Z, Silva AC, Yang J, Shen J (2005) Elevated endogenous GABA level correlates with decreased fMRI signals in the rat brain during acute inhibition of GABA transaminase. Journal of Neuroscience Research 79:383-391.

Cohen D (1968) Magnetoencephalography: Evidence of magnetic fields produced by alpharhyth currents. Science 161:784-786.

Conner CR, Ellmore TM, Pieters TA, DiSano MA, Tandon N (2011) Variability of the Relationship between Electrophysiology and BOLD-fMRI across Cortical Regions in Humans. The Journal of Neuroscience 31:12855-12865.

Cruetzfeldt OD (1975) Neurophysiological correlates of different functional states of the brain. Munksgaard.

Dagli MS, Ingeholm JE, Haxby JV (1999) Localization of Cardiac-Induced Signal Change in fMRI. NeuroImage 9:407-415.

Dale AM, Liu AK, Fischl BR, Buckner RL, Belliveau JW, Lewine JD, Halgren E (2000) Dynamic Statistical Parametric Mapping: Combining fMRI and MEG for High-Resolution Imaging of Cortical Activity. Neuron 26:55-67.

Damoiseaux JS, Rombouts SARB, Barkhof F, Scheltens P, Stam CJ, Smith SM, Beckmann CF (2006) Consistent resting-state networks across healthy subjects. Proceedings of the National Academy of Sciences 103:13848-13853.

de Pasquale F, Della Penna S, Snyder AZ, Lewis C, Mantini D, Marzetti L, Belardinelli P, Ciancetta L, Pizzella V, Romani GL, Corbetta M (2010) Temporal dynamics of spontaneous MEG activity in brain networks. Proceedings of the National Academy of Sciences 107:60406045.

Donahue MJ, Near J, Blicher JU, Jezzard P (2010) Baseline GABA concentration and fMRI response. NeuroImage 53:392-398.

Donner TH, Siegel M (2011) A framework for local cortical oscillation patterns. Trends in Cognitive Sciences 15:191-199.

Duncan NW, Enzi B, Wiebking C, Northoff G (2011) Involvement of glutamate in rest-stimulus interaction between perigenual and supragenual anterior cingulate cortex: A combined fMRI-MRS study. Human Brain Mapping 32:2172-2182.

Edden RAE, Muthukumaraswamy SD, Freeman TCA, Singh KD (2009) Orientation Discrimination Performance Is Predicted by GABA Concentration and Gamma Oscillation Frequency in Human Primary Visual Cortex. The Journal of Neuroscience 29:1572115726.

Engel AK, Singer W (2001) Temporal binding and the neural correlates of sensory awareness. Trends in Cognitive Sciences 5:16-25.

Falkenberg LE, Westerhausen R, Specht K, Hugdahl K (2012) Resting-state glutamate level in the anterior cingulate predicts blood-oxygen level-dependent response to cognitive control. Proceedings of the National Academy of Sciences 109:5069-5073.

Fujimaki N, Hayakawa T, Nielsen M, Knösche TR, Miyauchi S (2002) An fMRI-Constrained MEG Source Analysis with Procedures for Dividing and Grouping Activation. NeuroImage 17:324-343.

Gaetz W, Edgar JC, Wang DJ, Roberts TPL (2011) Relating MEG measured motor cortical oscillations to resting $\gamma$-Aminobutyric acid (GABA) concentration. NeuroImage 55:616621. 
Gauthier CJ, Madjar C, Tancredi FB, Stefanovic B, Hoge RD (2011) Elimination of visually evoked BOLD responses during carbogen inhalation: Implications for calibrated MRI. NeuroImage 54:1001-1011.

Goense JBM, Logothetis NK (2008) Neurohpysiology of the BOLD fMRI signal in awake monkeys. Current Biology 18:631-640.

Goloshevsky AG, Silva AC, Dodd SJ, Koretsky AP (2008) BOLD fMRI and somatosensory evoked potentials are well correlated over a broad range of frequency content of somatosensory stimulation of the rat forepaw. Brain Research 1195:67-76.

Gonçalves SI, de Munck JC, Pouwels PJW, Schoonhoven R, Kuijer JPA, Maurits NM, Hoogduin JM, Van Someren EJW, Heethaar RM, Lopes da Silva FH (2006) Correlating the alpha rhythm to BOLD using simultaneous EEG/fMRI: Inter-subject variability. NeuroImage 30:203213.

Gorodnitsky IF, George JS, Rao BD (1995) Neuromagnetic source imaging with FOCUSS: a recursive weighted minimum norm algorithm. Electroencephalography and Clinical Neurophysiology 95:231-251.

Gow DW, Segawa JA, Ahlfors SP, Lin FH (2008) Lexical influences on speech perception: a Granger causality analysis of MEG and EEG source estimates. NeuroImage 43:614-623.

Gross J, Kujala J, Hamalainen M, Timmermann L, Schnitzler A, Salmelin R (2001a) Dynamic imaging of coherent sources: Studying neural interactions in the human brain. Proceedings of the National Academy of Science USA 98:694-699.

Gross J, Kujala J, Hämäläinen M, Timmermann L, Schnitzler A, Salmelin R (2001b) Dynamic imaging of coherent sources: Studying neural interactions in the human brain. Proceedings of the National Academy of Sciences 98:694-699.

Gross J, Timmermann L, Kujala J, Dirks M, Schmitz F, Salmelin R, Schnitzler A (2002) The neural basis of intermittent motor control in humans. Proceedings of the National Academy of Science USA 99:2299-2302.

Grummich P, Nimsky C, Pauli E, Buchfelder M, Ganslandt O (2006) Combining fMRI and MEG increases the reliability of presurgical language localization: A clinical study on the difference between and congruence of both modalities. NeuroImage 32:1793-1803.

Hall EL, Woolrich MW, Thomaz CE, Morris PG, Brookes MJ (2013) Using variance information in magnetoencephalography measures of functional connectivity. NeuroImage 67:203-212.

Hall SD, Barnes GR, Furlong PL, Seri S, Hillebrand A (2010) Neuronal network pharmacodynamics of GABAergic modulation in the human cortex determined using pharmaco-magnetoencephalography. Human Brain Mapping 31:581-594.

Hall SD, Holliday IE, Hillebrand A, Singh KD, Furlong PL, Hadjipapas A, Barnes GR (2005) The missing link: analogous human and primate cortical gamma oscillations. NeuroImage 26:13-17.

Hall SD, Stanford IM, Yamawaki N, McAllister CJ, Rönnqvist KC, Woodhall GL, Furlong PL (2011) The role of GABAergic modulation in motor function related neuronal network activity. NeuroImage 56:1506-1510.

Hamalainen M, Hari R (2002) Magnetoencephalographic Characterization of Dynamic Brain Activation: Basic Principles and Methods of Data Collection and Source Analysis. In: Brain Mapping: The Methods(Toga, A. W. and Mazziotta, J. C., eds).

Hamalainen M, Hari R, Ilmoniemi RJ, Knuutila J, Lounasmaa OV (1993) MAGNETOENCEPHALOGRAPHY - THEORY, INSTRUMENTATION, AND APPLICATIONS TO NONINVASIVE STUDIES OF THE WORKING HUMAN BRAIN. Reviews of Modern Physics 65:413-497.

Hämäläinen MS, Ilmoniemi RJ (1994) Interpreting magnetic fields of the brain: minimum norm estimates. Med Biol Eng Comput 32:35-42.

Handwerker DA, Ollinger JM, D'Esposito M (2004) Variation of BOLD hemodynamic responses across subjects and brain regions and their effects on statistical analyses. NeuroImage 21:1639-1651.

Harvey BM, Vansteensel MJ, Ferrier CH, Petridou N, Zuiderbaan W, Aarnoutse EJ, Bleichner MG, Dijkerman HC, van Zandvoort MJE, Leijten FSS, Ramsey NF, Dumoulin SO (2013) 
Frequency specific spatial interactions in human electrocorticography: V1 alpha oscillations reflect surround suppression. NeuroImage 65:424-432.

Heidemann RM, Ivanov D, Trampel R, Fasano F, Meyer H, Pfeuffer J, Turner R (2012) Isotropic submillimeter fMRI in the human brain at $7 \mathrm{~T}$ : Combining reduced field-of-view imaging and partially parallel acquisitions. Magnetic Resonance in Medicine 68:1506-1516.

Henson RN, Flandin G, Friston KJ, Mattout J (2010) A Parametric Empirical Bayesian framework for fMRI-constrained MEG/EEG source reconstruction. Human Brain Mapping 31:15121531.

Hillebrand A, Barnes GR, Bosboom JL, Berendse HW, Stam CJ (2012) Frequency-dependent functional connectivity within resting-state networks: An atlas-based MEG beamformer solution. NeuroImage 59:3909-3921.

Hipp JF, Hawellek DJ, Corbetta M, Siegel M, Engel AK (2012) Large-scale cortical correlation structure of spontaneous oscillatory activity. Nat Neurosci 15:884-890.

Im C-H, Jung H-K, Fujimaki N (2005) fMRI-constrained MEG source imaging and consideration of fMRI invisible sources. Human Brain Mapping 26:110-118.

Ioannides AA, Liu LC, Kwapien J, Drozdz S, Streit M (2000) Coupling of regional activations in a human brain during an object and face affect recognition task. Human Brain Mapping 11:77-92.

Jerbi K, Lachaux JP, N'Diaye K, Pantazis D, Leahy RM, Garnero L, Baillet S (2007) Coherent neural representation of hand speed in humans revealed by MEG imaging. Proceedings of the National Academy of Science USA 104:7676-7681.

Jolles DD, van Buchem MA, Crone EA, Rombouts SARB (2011) A Comprehensive Study of WholeBrain Functional Connectivity in Children and Young Adults. Cerebral Cortex 21:385391.

Kwong KK, Belliveau JW, Chesler DA, Goldberg IE, Weisskoff RM, Poncelet BP, Kennedy DN, Hoppel BE, Cohen MS, Turner R, Cheng HM, Brady TJ, Rosen BR (1992) DYNAMIC MAGNETIC-RESONANCE-IMAGING OF HUMAN BRAIN ACTIVITY DURING PRIMARY SENSORY STIMULATION. Proc Natl Acad Sci U S A 89:5675-5679.

Liao Y, Tang J, Fornito A, Liu T, Chen X, Chen H, Xiang X, Wang X, Hao W (2012) Alterations in regional homogeneity of resting-state brain activity in ketamine addicts. Neuroscience Letters 522:36-40.

Liu H, Kale Edmiston E, Fan G, Xu K, Zhao B, Shang X, Wang F (2013a) Altered resting-state functional connectivity of the dentate nucleus in Parkinson's disease. Psychiatry Research: Neuroimaging 211:64-71.

Liu Y, Yu C, Zhang X, Liu J, Duan Y, Alexander-Bloch AF, Liu B, Jiang T, Bullmore E (2013b) Impaired Long Distance Functional Connectivity and Weighted Network Architecture in Alzheimer's Disease. Cerebral Cortex.

Liu Z, Fukunaga M, de Zwart JA, Duyn JH (2010) Large-scale spontaneous fluctuations and correlations in brain electrical activity observed with magnetoencephalography. NeuroImage 51:102-111.

Logothetis NK, Pauls J, Augath M, Trinath T, Oeltermann A (2001) Neurophysiological investigation of the basis of the fMRI signal. Nature 412:150-157.

Luckhoo H, Hale JR, Stokes MG, Nobre AC, Morris PG, Brookes MJ, Woolrich MW (2012) Inferring task-related networks using independent component analysis in magnetoencephalography. NeuroImage 62:530-541.

Moradi F, Liu LC, Cheng K, Waggoner RA, Tanaka K, Ioannides AA (2003) Consistent and precise localization of brain activity in human primary visual cortex by MEG and fMRI. NeuroImage 18:595-609.

Mosher JC, Lewis PS, Leahy RM (1992) Multiple dipole modeling and localization from spatiotemporal MEG data. Biomedical Engineering, IEEE Transactions on 39:541-557.

Mukamel R, Gelbard H, Arieli A, Hasson U, Fried I, Malach R (2005) Coupling Between Neuronal Firing, Field Potentials, and fMRI in Human Auditory Cortex. Science 309:951-954. 
Mullinger KJ, Mayhew SD, Bagshaw AP, Bowtell R, Francis ST (2013) Poststimulus undershoots in cerebral blood flow and BOLD fMRI responses are modulated by poststimulus neuronal activity. Proceedings of the National Academy of Sciences 110:13636-13641.

Muthukumaraswamy SD, Edden RAE, Jones DK, Swettenham JB, Singh KD (2009) Resting GABA concentration predicts peak gamma frequency and fMRI amplitude in response to visual stimulation in humans. Proceedings of the National Academy of Sciences 106:83568361.

Muthukumaraswamy SD, Evans CJ, Edden RAE, Wise RG, Singh KD (2012) Individual variability in the shape and amplitude of the BOLD-HRF correlates with endogenous GABAergic inhibition. Human Brain Mapping 33:455-465.

Muthukumaraswamy SD, Myers JFM, Wilson SJ, Nutt DJ, Lingford-Hughes A, Singh KD, Hamandi $\mathrm{K}$ (2013) The effects of elevated endogenous GABA levels on movement-related network oscillations. NeuroImage 66:36-41.

Muthukumaraswamy SD, Singh KD (2008) Spatiotemporal frequency tuning of BOLD and gamma band MEG responses compared in primary visual cortex. NeuroImage 40:15521560.

Muthukumaraswamy SD, Singh KD (2009) Functional decoupling of BOLD and gamma-band amplitudes in human primary visual cortex. Human Brain Mapping 30:2000-2007.

Niessing J, Ebisch B, Schmidt KE, Niessing M, Singer W, Galuske RAW (2005) Hemodynamic Signals Correlate Tightly with Synchronized Gamma Oscillations. Science 309:948-951.

Nolte G, Bai O, Wheaton L, Mari Z, Vorbach S, Hallett M (2004a) Identifying true brain interaction from EEG data using the imaginary part of coherency. Clinical Neurophysiology 115:2292 - 2307.

Nolte G, Bai O, Wheaton L, Mari Z, Vorbach S, Hallett M (2004b) Identifying true brain interaction from EEG data using the imaginary part of coherency. Clinical Neurophysiology 115:2292-2307.

Nolte G, Ziehe A, Nikulin VV, Schlögl A, Krämer N, Brismar T, Müller KR (2008) Robustly estimating the flow direction of information in complex physical systems. Physical Review Letters 100.

Northoff G, Walter M, Schulte RF, Beck J, Dydak U, Henning A, Boeker H, Grimm S, Boesiger P (2007) GABA concentrations in the human anterior cingulate cortex predict negative BOLD responses in fMRI. Nat Neurosci 10:1515-1517.

Ogawa S, Lee TM, Kay AR, Tank DW (1990) Brain magnetic resonance imaging with contrast dependent on blood oxygenation. Proceedings of the National Academy of Sciences 87:9868-9872.

Ogawa S, Tank DW, Menon R, Ellermann JM, Kim SG, Merkle H, Ugurbil K (1992) INTRINSIC SIGNAL CHANGES ACCOMPANYING SENSORY STIMULATION - FUNCTIONAL BRAIN MAPPING WITH MAGNETIC-RESONANCE-IMAGING. Proc Natl Acad Sci U S A 89:59515955.

Ou W, Nummenmaa A, Ahveninen J, Belliveau JW, Hämäläinen MS, Golland P (2010) Multimodal functional imaging using fMRI-informed regional EEG/MEG source estimation. NeuroImage 52:97-108.

Pascual-Marqui RD, Michel CM, Lehmann D (1994) Low resolution electromagnetic tomography: a new method for localizing electrical activity in the brain. International Journal of Psychophysiology 18:49-65.

Ramnania N, Behrens TEJ, Penny W, Matthews PM (2004) New approaches for exploring anatomical and functional connectivity in the human brain. Biological Psychiatry 56:613-619.

Robinson S, Vrba J (1998) Functional Neuroimaging by synthetic aperture magnetometry. Sendai: Tohoku University Press.

Robinson SE, Mandell AJ, Coppola R (2013) Spatiotemporal Imaging of Complexity. Frontiers in Computational Neuroscience 6. 
Roopun AK, Kramer MA, Carracedo LM, Kaiser M, Davies CH, Traub RD, Kopell NJ, Whittington MA (2008) Temporal interactions between cortical rhythms. Frontiers in Neuroscience 2.

Rossini PM, Altamura C, Ferretti A, Vernieri F, Zappasodi F, Caulo M, Pizzella V, Del Gratta C, Romani GL, Tecchio F (2004) Does cerebrovascular disease affect the coupling between neuronal activity and local haemodynamics? Brain 127:99-110.

Sato M-a, Yoshioka T, Kajihara S, Toyama K, Goda N, Doya K, Kawato M (2004) Hierarchical Bayesian estimation for MEG inverse problem. Neurolmage 23:806-826.

Schlögl A, Supp G (2006) Analyzing event-related EEG data with multivariate autoregressive parameters.Schlögl A, Supp G. Prog Brain Res 159:135-147.

Schoffelen JM, Gross J (2009) Source connectivity analysis with MEG and EEG. Human Brain Mapping 30:1857-1865.

Schölvinck ML, Leopold DA, Brookes MJ, Khader PH (2013) The contribution of electrophysiology to functional connectivity mapping. Neurolmage 80:297-306.

Seeley WW, Crawford RK, Zhou J, Miller BL, Greicius MD (2009) Neurodegenerative Diseases Target Large-Scale Human Brain Networks. Neuron 62:42-52.

Sekihara K, Nagarajan SS, Peoppel D, Marantz A, Miyashita Y (2001) Reconstructing SpatioTemporal Activities of Neural Sources Using an MEG Vector Beamformer Technique. IEEE Transactions on Biomedical Engineering 48:760-771.

Sekihara K, Owen JP, Trisno S, Nagarajan SS (2011) Removal of Spurious Coherence in MEG Source-Space Coherence Analysis. Biomedical Engineering, IEEE Transactions on 58:3121-3129.

Shmuel A, Augath M, Oeltermann A, Logothetis NK (2006) Negative functional MRI response correlates with decreases in neuronal activity in monkey visual area V1. Nat Neurosci 9:569-577.

Singh KD (2006) Magnetoencephalography. In: Methods in Mind(Senior, C. et al., eds): MIT Press.

Singh KD, Barnes GR, Hillebrand A, Forde EME, Williams AL (2002) Task-Related Changes in Cortical Synchronization Are Spatially Coincident with the Hemodynamic Response. NeuroImage 16:103-114.

Stam CJ, Nolte G, Daffertshofer A (2007) Phase lag index: Assessment of functional connectivity from multi channel EEG and MEG with diminished bias from common sources. Human Brain Mapping 28:1178-1193.

Stam CJ, van Dijk BW (2002) Synchronization likelihood: an unbiased measure of generalized synchronization in multivariate data sets. Physica D: Nonlinear Phenomena 163:236251.

Stevenson CM, Brookes MJ, Morris PG (2011) $\beta$-Band correlates of the fMRI BOLD response. Human Brain Mapping 32:182-197.

Stevenson CM, Wang F, Brookes MJ, Zumer JM, Francis ST, Morris PG (2012) Paired pulse depression in the somatosensory cortex: Associations between MEG and BOLD fMRI. NeuroImage 59:2722-2732.

Swettenham JB, Muthukumaraswamy SD, Singh KD (2013) BOLD responses in human primary visual cortex are insensitive to substantial changes in neural activity. Frontiers in Human Neuroscience 7.

Tass P, Rosenblum MG, Weule J, Kurths J, Pikovsky A, Volkmann J, Schnitzler A, Freund HJ (1998) Detection of $n: m$ Phase Locking from Noisy Data: Application to Magnetoencephalography. Physical Review Letters 81:3291-3294.

Tian L, Jiang T, Wang Y, Zang Y, He Y, Liang M, Sui M, Cao Q, Hu S, Peng M, Zhuo Y (2006) Altered resting-state functional connectivity patterns of anterior cingulate cortex in adolescents with attention deficit hyperactivity disorder. Neuroscience Letters 400:39-43.

Timmermann L, Gross J, Dirks M, Volkmann J, Freund HJ, Schnitzler A (2002) The cerebral oscillatory network of parkinsonian resting tremor. Brain 126:199-212. 
Tootell RBH, Reppas JB, Kwong KK, Malach R, Born RT, Brady TJ, Rosen BR, Belliveau JW (1995) Functional analysis of human MT and related visual cortical areas using magnetic resonance imaging. The Journal of Neuroscience 15:3215-3230.

Van Drongelen W, Yuchtman M, Van Veen BD, Van Huffelen AC (1996) A Spatial Filtering Technique to Detect and Localize Multiple Sources in the Brain. Brain Topography 9:3949.

Van Veen BD, Van Drongelen W, Yuchtman M, Suzuki A (1997) Localisation of brain electrical activity via linearly constrained minimum variance spatial filtering. IEEE Transactions on biomedical engineering 44:867-880.

Vrba J, Robinson SE (2001) Signal Processing in Magnetoencephalography. Methods 25:249271.

Winterer G, Carver FW, Musso F, Mattay V, Weinberger DR, Coppola R (2007) Complex relationship between BOLD signal and synchronization/desynchronization of human brain MEG oscillations. Human Brain Mapping 28:805-816.

Wipf DP, Owen JP, Attias HT, Sekihara K, Nagarajan SS (2010) Robust Bayesian estimation of the location, orientation, and time course of multiple correlated neural sources using MEG. NeuroImage 49:641-655.

Zhu Z, Zumer JM, Lowenthal ME, Padberg J, Recanzone GH, Krubitzer LA, Nagarajan SS, Disbrow EA (2009) The relationship between magnetic and electrophysiological responses to complex tactile stimuli. BMC Neuroscience 10.

Zumer JM, Brookes MJ, Stevenson CM, Francis ST, Morris PG (2010) Relating BOLD fMRI and neural oscillations through convolution and optimal linear weighting. NeuroImage 49:1479-1489. 
Table 1: Spatial relationship between BOLD fMRI and MEG responses

\begin{tabular}{|c|c|c|c|}
\hline Author & Stimulus & MEG Signal & $\begin{array}{l}\text { Separation } \\
(\mathrm{mm})\end{array}$ \\
\hline \multirow{2}{*}{ (Stevenson et al., 2011) } & \multirow{2}{*}{ Visual } & $\beta$-ERD & $9 \pm 2$ \\
\hline & & $\beta$-ERS & $14 \pm 2$ \\
\hline \multirow{2}{*}{ (Stevenson et al., 2012) } & \multirow{2}{*}{ Median nerve } & $\beta$-ERD & $29 \pm 6$ \\
\hline & & $\beta$-ERS & $13 \pm 2$ \\
\hline \multirow{6}{*}{ (Winterer et al., 2007) } & \multirow{6}{*}{$\begin{array}{l}\text { Visual, button } \\
\text { press to indicate } \\
\text { LHS/RHS stimulus }\end{array}$} & $\alpha$-ERD, L visual & 16.2 \\
\hline & & $\alpha$-ERD, R parietal & 10.3 \\
\hline & & $\beta$-ERD, L motor & 21.8 \\
\hline & & $\gamma$-ERD, L motor & 16.9 \\
\hline & & $\theta$-ERD, R motor & 24.1 \\
\hline & & $\theta$-ERD, R ACC & 28.7 \\
\hline \multirow{3}{*}{ (Brookes et al., 2005) } & \multirow{3}{*}{ Visual } & sustained field & $8 \pm 12$ \\
\hline & & $\gamma$-ERD & $9 \pm 15$ \\
\hline & & $\alpha$-ERD & $11 \pm 9$ \\
\hline $\begin{array}{l}\text { (Muthukumaraswamy and } \\
\text { Singh, 2008) }\end{array}$ & Visual & $\gamma$ & $6 \pm 1$ \\
\hline (Moradi et al., 2003) & Visual & Evoked field & $3 \pm 2$ \\
\hline (Zumer et al., 2010) & Visual & $\beta$-ERD $/ \gamma$-ERS & $13 \pm 3$ \\
\hline
\end{tabular}




\section{Figure Captions}

Figure 1: Schematic diagram showing the origins of both the MEG and BOLD signals. From an initial stimulus a neuronal response is generated which induces a magnetic field and a vascular response via chemical and metabolic signalling. The magnetic fields generated by the neuronal response are detected in MEG. The changes in the deoxyhaemaglobin levels ( $\mathrm{dHb}$ ) due to vascular changes (blood flow (CBF), volume (CBV) and oxygen consumption $\left(\mathrm{CMRO}_{2}\right)$ are detected in BOLD fMRI.

Figure 2: Spatial relationship between MEG and fMRI for a letter fluency task. (a) is the group fMRI results and (b) is the group beamformer image (peak across all frequency bands is shown for each voxel). Figure reproduced from Singh et al. 2002 Task-Related Changes in Cortical Synchronization Are Spatially Coincident with the Hemodynamic Response. NeuroImage 16:103114

Figure 3: a) Timecourses of neural oscillatory amplitude in visual cortex in response to a simple visual stimulus. Different colours correspond to different frequency bands. b) Correlation between deconvolved BOLD and MEG responses, plotted as a function of frequency c) Equivalent correlation, measured invasively in human auditory cortex in epilepsy patients undergoing natural stimulation (watching a movie). Figures $3 \mathrm{a}$ and $3 \mathrm{~b}$ reproduced from Zumer et al. 2010 Relating BOLD fMRI and neural oscillations through convolution and optimal linear weighting. NeuroImage 49:1479-1489. Figure 3c reproduced from Mukamel et al. 2005 Coupling Between Neuronal Firing, Field Potentials, and fMRI in Human Auditory Cortex. Science 309:951-954.

Figure 4: (i) The spatial relationship between fMRI and MEG measures of functional networks. (ii) Frequency dependence of correlation between two notes of the sensorimotor network. Real data is shown in red, matched simulated data in green, and the blue shaded region indicates the 95\% confidence interval, Figures adapted from Brookes et al. 2011 Investigating the electrophysiological basis of resting state networks using magnetoencephalography Proc Natl Acad Science 108:16783-16788.

Figure 5: The relationship between GABA, stimulus induced peak gamma frequency and BOLD change. Figure reproduced from Muthukuraswamy et al (2009) Resting GABA concentration 
predicts peak gamma frequency and fMRI amplitude in response to visual stimulation in humans, Proc Natl Acad Science 106:8356-8361 